\title{
Central obesity is associated with helicobacter pylori infection: a large-scale cross-sectional retrospective study in West China
}

\author{
Qinqin $\mathrm{Wu}^{1} \cdot$ Ken $\mathrm{Qin}^{1} \cdot$ Youjuan Wang ${ }^{1}$ (D)
}

Received: 12 March 2019 / Accepted: 29 July 2019 /Published online: 14 August 2019

(C) The Author(s) 2019

\begin{abstract}
Context The association between obesity and Helicobacter pylori (H. pylori) infection remains controversial.

Aims The objective is to investigate the relationship between obesity and $H$. pylori infection, as diagnosed on the basis of a $14 \mathrm{C}$ urea breath test (14C-UBT) and waist circumference (WC).

Settings and design A retrospective cross-sectional study was performed at Health management center of a Tertiary care teaching hospital in Southwest of China.

Materials and methods Clinical information of 76,915 individuals (46,003 men and 30,912 women) with 44.0 (35.0-51.0) years was extracted from medical record. H. pylori infection was diagnosed by a positive 14C-UBT, and obesity was defined as WC $\geq$ $90 \mathrm{~cm}$ in men and $\geq 80 \mathrm{~cm}$ in women.

Statistical analysis Descriptive statistics, Student's $t$ test, Mann-Whitney $U$ test, and chi-square test, followed by binary logistic regression were performed in SPSS.

Results The overall prevalence of $H$. pylori infection was $39.95 \%$. The prevalence of $H$. pylori infection in subjects with central obesity was significantly higher than that in normal-WC subjects $(42.20 \%$ vs. $39.10 \%, p<0.001)$. WC was significantly higher in H. pylori-positive subjects compared with $H$. pylori-negative subjects $(p<0.01)$. There was a linear association between WC quintiles and $H$. pylori infection prevalence. After adjusting for confounders, central obesity was associated with $H$. pylori infection $(\mathrm{OR}=1.052,95 \% \mathrm{CI}, 1.009-1.096, p=0.02)$.

Conclusions Central obesity is associated with H. pylori infection after adjusting for multiple confounding factors such as age, gender, and lifestyle characteristics.
\end{abstract}

Keywords Helicobacter pylori $\cdot$ Central obesity $\cdot{ }^{14} \mathrm{C}$ urea breath test $\cdot$ Waist circumference

\section{Background}

Helicobacter pylori (H. pylori) infection affects almost half of the world's population. H. pylori infection contributes to a variety of gastrointestinal diseases, such as chronic gastritis, peptic ulcer, gastric cancer, and gastric mucosa-associated lymphoma (MALT). Additionally, H. pylori infection also plays a role in extragastric diseases, such as cardiovascular and immunological systemic disorders. Recently, chronic spontaneous urticaria and insulin resistance [1] have also been found to be associated with $H$. pylori infection. On the other

Qinqin $\mathrm{Wu}$

wuqinqin1028@hotmail.com

1 Department of Health Management Center, West China Hospital, Sichuan University, Add: No. 37 Guo Xue Xiang, Chengdu 610041, Sichuan, People's Republic of China hand, obesity is also a growing global health problem. But the relationship between $H$. pylori infection and obesity remains controversial [2-7]. Some studies identified a positive correlation [3, 8-10], while others found a negative correlation [4, 7] or even no correlation [2, 11]. A meta-analysis of 18 observational studies involving over 10,000 subjects found a higher body mass index (BMI) among H. pylori-positive subjects compared with $H$. pylori-negative subjects [12]. It is known that BMI has significant limitations in the assessment of obesity $[6,13]$. Obesity can be assessed by several methods such as BMI and waist circumference (WC). In the majority of prior studies that explored the association between $H$. pylori infection and obesity, obesity was defined by BMI. For the first time, we used WC to define obesity to evaluate such a relationship. Both BMI and WC are commonly used to estimate body fat. But BMI does not distinguish fat mass from lean mass, nor does it necessarily reflect body fat distribution, particularly in those with BMI $<30 \mathrm{~kg} / \mathrm{m}^{2}$ [14]. When used in 
older people or muscular individuals such as athletes, BMI may lead to an inappropriate diagnosis of obesity, because it generally overestimates adiposity on those with more lean body mass [14]. ${ }^{14} \mathrm{C}$ urea breath test $\left({ }^{14} \mathrm{C}\right.$-UBT $)$ was a simple invasive methods with high sensitivity 0.96 (95\% CI $0.95-$ 0.97 ) and specificity 0.93 (95\% CI $0.91-0.94$ ) to detect H. pylori infection [15]. In order to explore the association between $H$. pylori infection diagnosed by ${ }^{14} \mathrm{C}$-UBT and obesity diagnosed on the basis of WC, we conducted a large-scale cross-sectional retrospective study in West China.

\section{Methods}

\section{Data collection}

Subjects who underwent ${ }^{14} \mathrm{C}$-UBT in the Health Management Center of West China Hospital were enrolled into the study from December 2013 to December 2014. The analyses were limited to the participants who had complete records of anthropometric, biochemical data and had results of $H$. pylori ${ }^{14} \mathrm{C}$ UBT test. A total of 76,915 individuals (46,003 men and 30,912 women) with a medium (interquartile ranges) age of 44.0 (35.0-51.0) years were involved in the final analysis. All subjects had the data about their medical history (hypertension and diabetes mellitus), smoking (current smoking defined as $\geq 20$ cigarettes per month for $\geq 6$ months) and alcohol use (current alcohol use defined as at least once per week for $\geq 6$ months).

The measurement of WC, body weight, blood pressure, height, and hip circumference was recorded and documented. The measurement was performed by trained nurses. The participants were in a standing position with light clothes. WC was measured at the level of the iliac crest. All participants were asked to stand upright with arms hanging freely in normal clothing without shoes. All measurements were measured using the non-elastic tape on bare skin. WC was measured horizontally at the midpoint between the last rib and the iliac crest to the nearest $0.1 \mathrm{~cm}$ [16]. The average of three measurements was taken. Hip circumference was measured at the broadest part of the buttock. Height was measured by an inelastic ruler to the nearest $0.5 \mathrm{~cm}$. Weight was measured to the nearest $0.1 \mathrm{~kg}$ in light clothing and without shoes using Weight scale (Wujin, RGT-120, Wujin Medical Equipment Co. Ltd., Jiangsu, China). BMI was calculated as weight/height ${ }^{2}\left(\mathrm{~kg} / \mathrm{m}^{2}\right)$. Blood pressure was measured with the sitting position by a sphygmomanometer (Yuyue, GB 3053-93, Yuyue Medical Equipment Co., Ltd., Jiangsu, China) on the right arm after a 5 -min rest. The mean of two measurements at least $1 \mathrm{~min}$ apart was recorded.

\section{Diagnosis of $H$. pylori infection}

H. pylori infection was diagnosed by a positive ${ }^{14} \mathrm{C}-\mathrm{UBT}$. To avoid false-negative results, subjects were asked not to take any antibiotics for at least 4 weeks or any proton pump inhibitors or $\mathrm{H}_{2}$ antagonists for at least 1-2 weeks before the test. The subjects took a ${ }^{14} \mathrm{C}$-labeled urea containing tablet (Shanghai Xinke Pharmaceutical Co., Ltd., Shanghai, China) with $120-\mathrm{mL}$ water after an overnight fast. The dose of radiation was as small as $1 \mu \mathrm{Ci}$. After $15 \mathrm{~min}$, the subject breathed out into a dry breath card (Anhui Yanghe Pharmaceutical Co., Ltd., Anhui, China) until the color of the card indicator changed from blue to white, which took about 2-5 min. Thereafter, the card was inserted into the YH04D machine (Anhui Yanghe Pharmaceutical Co., Ltd., Anhui, China) to detect the presence of $H$. pylori. Results were labeled as H. pylori positive or negative.

\section{Diagnosis of obesity}

Obesity was assessed according to waist circumference. For men, a WC of $<90 \mathrm{~cm}$ was defined as normal WC, and a WC $\geq 90 \mathrm{~cm}$ as central obesity. For women, a WC $<80 \mathrm{~cm}$ was defined as normal $\mathrm{WC}$, and a $\mathrm{WC} \geq 80 \mathrm{~cm}$ as central obesity [17]. All the subjects were classified into quintiles according to their WC: $\mathrm{WC} \leq 73 \mathrm{~cm}, 73-81 \mathrm{~cm}, 81-88 \mathrm{~cm}$, and $\geq 88 \mathrm{~cm}$ for quintile $1,2,3$, and 4 , respectively.

\section{Blood test}

Blood samples were collected from the antecubital vein after an overnight fast and were analyzed without freezing. The samples were centrifuged at $3000 \mathrm{rpm}$ for $10 \mathrm{~min}$, and then analyzed. Fasting plasma glucose (FPG), total cholesterol (TC), high-density lipoproteins cholesterol (HDL-C), triglycerides (TG), alanine aminotransferase (ALT), aspartate aminotransferase (AST), $\gamma$-glutamyltransferase $(\gamma$-GGT), serum uric acid (UA), serum creatinine (Scr), and blood urea nitrogen (BUN) were analyzed by a biochemical autoanalyzer (ROCHE cobas 8000, ROCHE Diagnostics, Basel, Switzerland) at the Department of Clinical Laboratory Diagnostics, West China Hospital Sichuan University. Plasma glucose concentrations were assayed by the hexokinase method. TGs in plasma were assayed by means of glycerol oxidation. TC was measured by enzymic method. ALT, AST, and $\gamma$-GGT were assayed by means of the enzyme rate method. UA, Scr, and BUN were assayed by means of enzyme coupling method. HDL-C was measured by homogeneous enzyme colorimetry. Low-density lipoproteins cholesterol (LDL-C) was calculated using the Friedewald formula [18], i.e., LDL-C $=$ TC $-($ HDL-C $)-(T G / 5)$.

\section{Statistical analysis}

All tests were performed using the IBM SPSS Statistics 19.0 (IBM Corp, New York, NY, USA). Data normality was tested by Kolmogorov-Smirnov test. Continuous variables were 
presented as mean \pm standard deviation for normal distributions, and medians (25th to 75th percentiles) for skewed distributions. Categorical variables were presented as percentage (\%). Statistical analysis of continuous variables was performed using the Student's $t$ test and Mann-Whitney $U$ test, while analysis of categorical variables were performed using the chi-squared test. Binary logistic regression models were used to explore the correlation between central obesity and H. pylori infection after adjustment for a variety of subject's characteristics. The odds ratio (OR) and $95 \%$ confidence interval $(95 \% \mathrm{CI})$ were calculated. A two-tailed $p$ value of $<0.05$ was considered statistically significant.

\section{Results}

\section{Clinical and demographic characteristics}

A total of 76,915 subjects $(46,003$ men [mean age, $45.19 \pm$ 12.52 years] and 30,912 women [mean age, 43.14 \pm
12.39 years]) were enrolled into this cross-sectional retrospective study (Table 1) The overall prevalence of $H$. pylori infection was $39.95 \%, 39.10 \%$ in normal-WC subjects, and $42.20 \%$ in subjects with central obesity, respectively $\left(\chi^{2}=61.613, \mathrm{OR}=1.138,95 \% \mathrm{CI}, 1.102-1.175\right)$.

The characteristics of subjects with normal WC and central obesity are summarized in Table 1 . The prevalence of H. pylori infection in subjects with central obesity was significantly higher than that in normal-WC subjects $(42.20 \%$ vs. $39.10 \%, p<0.001)$. Other parameters including age, height, BMI, WC, hip circumference (HC), systolic blood pressure (SBP), diastolic blood pressure (DBP), pulse pressure(PP), fasting blood glucose (FPG), LDL-C, triglycerides (TG), total cholesterol (TC), uric acid (UA), serum creatinine (Scr), blood urea nitrogen (BUN), aminotransferase (ALT), $\gamma$ glutamyltransferase ( $\gamma$-GGT), aspartate aminotransferase(AST), and prevalence of smoking, alcohol use, hypertension, and diabetes mellitus were significantly higher, but the HDL-C level was significantly lower in subjects with central obesity compared with normal-WC subjects (all $p<0.01$ ).

Table 1 Comparison of clinical and demographic characteristics between subjects with normal WC and central obesity

\begin{tabular}{|c|c|c|c|c|c|c|}
\hline & \multicolumn{2}{|l|}{ Total $n=76,915$} & \multicolumn{2}{|l|}{ Male $n=46,003$} & \multicolumn{2}{|c|}{ Female $n=30,912$} \\
\hline & $\begin{array}{l}\text { Normal WC } \\
n=55,839\end{array}$ & $\begin{array}{l}\text { Central obesity } \\
n=21,076\end{array}$ & $\begin{array}{l}\text { Normal WC } \\
n=32,048\end{array}$ & $\begin{array}{l}\text { Central obesity } \\
n=13,955\end{array}$ & $\begin{array}{l}\text { Normal WC } \\
n=23,791\end{array}$ & $\begin{array}{l}\text { Central obesity } \\
n=7121\end{array}$ \\
\hline Age (years) & $42.92 \pm 12.31$ & $48.19 \pm 12.21$ & $44.49 \pm 12.76$ & $46.79 \pm 11.8$ & $40.8 \pm 11.35$ & $50.95 \pm 12.52$ \\
\hline Height $(\mathrm{cm})$ & $163.05 \pm 7.56$ & $164.78 \pm 8.47$ & $167.31 \pm 5.95$ & $169.03 \pm 6.05$ & $157.31 \pm 5.4$ & $156.43 \pm 5.92$ \\
\hline BMI $\left(\mathrm{kg} / \mathrm{m}^{2}\right)$ & $22.49 \pm 2.6$ & $26.93 \pm 2.71$ & $23.55 \pm 2.42$ & $27.69 \pm 2.38$ & $21.07 \pm 2.13$ & $25.45 \pm 2.71$ \\
\hline Waist circumference $(\mathrm{cm})$ & $76.63 \pm 7.75$ & $91.69 \pm 6.58$ & $81.27 \pm 5.84$ & $94.82 \pm 4.66$ & $70.38 \pm 5.19$ & $85.57 \pm 5.38$ \\
\hline Hip circumference (cm) & $91.65 \pm 4.9$ & $99.17 \pm 5.31$ & $93.26 \pm 4.57$ & $100.7 \pm 4.62$ & $89.48 \pm 4.48$ & $96.18 \pm 5.3$ \\
\hline Systolic blood pressure (mmHg) & $111.16 \pm 14.71$ & $120.38 \pm 16.2$ & $114.66 \pm 14.44$ & $121.62 \pm 15.31$ & $106.46 \pm 13.72$ & $117.95 \pm 17.55$ \\
\hline Diastolic blood pressure $(\mathrm{mmHg})$ & $72.81 \pm 9.46$ & $78.29 \pm 10.46$ & $75.21 \pm 9.48$ & $80.13 \pm 10.33$ & $69.57 \pm 8.4$ & $74.71 \pm 9.75$ \\
\hline Pulse pressure (mmHg) & $38.36 \pm 10.35$ & $42.09 \pm 11.94$ & $39.44 \pm 10.69$ & $41.5 \pm 11.34$ & $36.89 \pm 9.7$ & $43.25 \pm 12.97$ \\
\hline Fasting blood glucose $(\mathrm{mmol} / \mathrm{L})$ & $5.21 \pm 1.01$ & $5.65 \pm 1.47$ & $5.33 \pm 1.2$ & $5.74 \pm 1.6$ & $5.04 \pm 0.65$ & $5.48 \pm 1.15$ \\
\hline Triglycerides $(\mathrm{mmol} / \mathrm{L})$ & $1.45 \pm 1.17$ & $2.12 \pm 1.67$ & $1.72 \pm 1.34$ & $2.38 \pm 1.84$ & $1.09 \pm 0.74$ & $1.62 \pm 1.1$ \\
\hline LDL-cholesterol (mmol/L) & $2.78 \pm 0.77$ & $2.99 \pm 0.79$ & $2.9 \pm 0.77$ & $3 \pm 0.78$ & $2.61 \pm 0.74$ & $2.98 \pm 0.79$ \\
\hline HDL cholesterol $(\mathrm{mmol} / \mathrm{L})$ & $1.55 \pm 0.41$ & $1.32 \pm 0.34$ & $1.4 \pm 0.36$ & $1.22 \pm 0.28$ & $1.74 \pm 0.38$ & $1.53 \pm 0.36$ \\
\hline Total cholesterol $(\mathrm{mmol} / \mathrm{L})$ & $4.78 \pm 0.89$ & $5 \pm 0.94$ & $4.85 \pm 0.9$ & $5 \pm 0.93$ & $4.67 \pm 0.87$ & $5.01 \pm 0.95$ \\
\hline Serum uric acid $(\mu \mathrm{mol} / \mathrm{L})$ & $336.51 \pm 87.67$ & $379.04 \pm 93.36$ & $384.93 \pm 75.58$ & $417.08 \pm 81.74$ & $271.28 \pm 54.15$ & $304.48 \pm 65.66$ \\
\hline Creatinine $(\mu \mathrm{mol} / \mathrm{L})$ & $76.17 \pm 16.2$ & $77.99 \pm 16.16$ & $85.93 \pm 12.65$ & $85.31 \pm 13.19$ & $63.02 \pm 9.95$ & $63.64 \pm 10.99$ \\
\hline Blood urea nitrogen $(\mathrm{mmol} / \mathrm{L})$ & $4.97 \pm 1.29$ & $5.18 \pm 1.31$ & $5.22 \pm 1.29$ & $5.28 \pm 1.29$ & $4.63 \pm 1.2$ & $5 \pm 1.32$ \\
\hline Alanine aminotransferase (IU/L) & $25.01 \pm 18.06$ & $35.2 \pm 24.66$ & $30.38 \pm 20.18$ & $40.71 \pm 26.06$ & $17.77 \pm 11.21$ & $24.38 \pm 17.09$ \\
\hline Aspartate aminotransferase (IU/L) & $145.58 \pm 20.44$ & $148.83 \pm 20.78$ & $155.08 \pm 18.46$ & $156.5 \pm 18.92$ & $132.78 \pm 15.37$ & $133.8 \pm 15.35$ \\
\hline$\gamma$-Glutamyltransferase (IU/L) & $28.58 \pm 41.07$ & $45.84 \pm 55.49$ & $37.27 \pm 49.78$ & $55.81 \pm 61.39$ & $16.87 \pm 19.53$ & $26.31 \pm 33.95$ \\
\hline H. pylori infection (\%) & 39.10 & 42.20 & 39.60 & 42.20 & 38.50 & 42.30 \\
\hline Smoking (\%) & 30.00 & 37.50 & 51.20 & 55.90 & 1.40 & $1.60 *$ \\
\hline Alcohol use (\%) & 45.30 & 53.70 & 72.80 & 76.80 & 8.40 & $8.60 *$ \\
\hline Hypertension (\%) & 5.10 & 14.60 & 6.70 & 14.70 & 3.00 & 14.40 \\
\hline Diabetes mellitus (\%) & 2.20 & 4.80 & 3.10 & 5.30 & 0.80 & 3.90 \\
\hline
\end{tabular}

$* p>0.05$ 
As shown in Table 2, parameters including age, height, BMI, WC, HC, SBP, DBP, PP, FPG, LDL-C, TG, TC, UA, Scr, BUN, ALT, $\gamma$-GGT, AST, and prevalence of smoking, alcohol use, hypertension, and diabetes mellitus were significantly higher, but the HDL-C level was significantly lower in the $H$. pylori-positive subjects with central obesity compared with $H$. pylori-positive and normal-WC subjects in both total and male subjects (all $p<0.01$ ), except alcohol use, smoking, and creatinine in female $(p>0.05)$. H. pylori-positive with central obesity participants were related to a more bad metabolic profile than $H$. pylori-positive and normal-WC subjects.

The characteristics of H. pylori-positive and H. pylori-negative subjects are shown in Table 3. Multiple parameters including age, WC, HC, SBP, DBP, FBG, TG, LDL-C, TC, UA, creatinine, and prevalence of central obesity, smoking, alcohol use, hypertension, and diabetes mellitus were significantly higher, but the HDL-C level was significantly lower in H. pylori-positive subjects compared with $H$. pylori-negative subjects (all $p<0.01$ ). Height, PP, ALT, AST, $\gamma$-GGT, and BUN were comparable between $H$. pylori-positive and
H. pylori-negative subjects. Parameters including age, height, WC, HC, SBP, DBP, FBG, LDL-C, TC, UA, Scr, AST, and prevalence of central obesity, smoking, hypertension, and diabetes mellitus were significantly higher, but the HDL-C level was significantly lower in $H$. pylori-positive subjects compared with $H$. pylori-negative subjects (all $p<0.01)$. Height, PP, ALT, $\gamma$-GGT, TG, alcohol use, and BUN were comparable between $H$. pylori-positive and H. pylori-negative subjects in male. Characteristics including age, height, WC, HC, FBG, LDL-C, TC, AST, ALT, TG and prevalence of central obesity, smoking, hypertension, and diabetes mellitus were significantly higher in $H$. pylori-positive subjects compared with $H$. pylori-negative subjects (all $p<0.01)$. SBP, DBP, PP, $\gamma$-GGT, HDL-C, UA, Scr, hypertension, and BUN were comparable between $H$. pylori-positive and $H$. pylori-negative subjects in female.

A linear association between WC quintiles and H. pylori infection prevalence was detected. The prevalence was $37.76 \%$ among the subjects with $\mathrm{WC}$ in the first quintile and increased to $39.48 \%, 40.77 \%$, and

Table 2 The association between H. pylori-positive with normal WC and H. pylori-positive with central obesity

\begin{tabular}{|c|c|c|c|c|c|c|}
\hline & \multicolumn{2}{|c|}{$\begin{array}{l}\text { Total H. pylori-positive } \\
n=30,726\end{array}$} & \multicolumn{2}{|c|}{$\begin{array}{l}\text { Male } H . \text { pylori-positive } \\
n=18,559\end{array}$} & \multicolumn{2}{|c|}{$\begin{array}{l}\text { Female } H . \text { pylori-positive } \\
n=12,167\end{array}$} \\
\hline & $\begin{array}{l}\text { Normal WC } \\
n=21,831\end{array}$ & $\begin{array}{l}\text { Central obesity } \\
n=8895\end{array}$ & $\begin{array}{l}\text { Normal WC } \\
n=12,676\end{array}$ & $\begin{array}{l}\text { Central obesity } \\
n=5883\end{array}$ & $\begin{array}{l}\text { Normal WC } \\
n=9155\end{array}$ & $\begin{array}{l}\text { Central obesity } \\
n=3012\end{array}$ \\
\hline Age (years) & $45.31 \pm 12.59$ & $47.23 \pm 11.55$ & $45.31 \pm 12.59$ & $47.23 \pm 11.55$ & $41.88 \pm 11.16$ & $50.95 \pm 11.91$ \\
\hline Height (cm) & $167.16 \pm 5.9$ & $168.97 \pm 5.97$ & $167.16 \pm 5.9$ & $168.97 \pm 5.97$ & $157.17 \pm 5.41$ & $156.6 \pm 5.82$ \\
\hline BMI $\left(\mathrm{kg} / \mathrm{m}^{2}\right)$ & $23.58 \pm 2.41$ & $27.75 \pm 2.37$ & $23.58 \pm 2.41$ & $27.75 \pm 2.37$ & $21.12 \pm 2.14$ & $25.46 \pm 2.66$ \\
\hline Waist circumference $(\mathrm{cm})$ & $81.42 \pm 5.76$ & $94.93 \pm 4.74$ & $81.42 \pm 5.76$ & $94.93 \pm 4.74$ & $70.52 \pm 5.18$ & $85.52 \pm 5.2$ \\
\hline Hip circumference $(\mathrm{cm})$ & $93.22 \pm 4.54$ & $100.7 \pm 4.64$ & $93.22 \pm 4.54$ & $100.7 \pm 4.64$ & $89.5 \pm 4.43$ & $96.28 \pm 5.32$ \\
\hline Systolic blood pressure (mmHg) & $115 \pm 14.59$ & $121.69 \pm 15.22$ & $115 \pm 14.59$ & $121.69 \pm 15.22$ & $106.44 \pm 13.8$ & $117.73 \pm 17.39$ \\
\hline Diastolic blood pressure (mmHg) & $75.51 \pm 9.58$ & $80.16 \pm 10.27$ & $75.51 \pm 9.58$ & $80.16 \pm 10.27$ & $69.53 \pm 8.46$ & $74.78 \pm 9.77$ \\
\hline Pulse pressure (mmHg) & $39.49 \pm 10.69$ & $41.53 \pm 11.22$ & $39.49 \pm 10.69$ & $41.53 \pm 11.22$ & $36.91 \pm 9.79$ & $42.95 \pm 12.79$ \\
\hline Fasting blood glucose $(\mathrm{mmol} / \mathrm{L})$ & $5.35 \pm 1.29$ & $5.75 \pm 1.6$ & $5.35 \pm 1.29$ & $5.75 \pm 1.6$ & $5.05 \pm 0.64$ & $5.49 \pm 1.18$ \\
\hline Triglycerides (mmol/L) & $1.72 \pm 1.36$ & $2.38 \pm 1.82$ & $1.72 \pm 1.36$ & $2.38 \pm 1.82$ & $1.09 \pm 0.8$ & $1.63 \pm 1.14$ \\
\hline LDL-cholesterol (mmol/L) & $2.92 \pm 0.78$ & $3.01 \pm 0.78$ & $2.92 \pm 0.78$ & $3.01 \pm 0.78$ & $2.65 \pm 0.74$ & $2.98 \pm 0.8$ \\
\hline HDL cholesterol (mmol/L) & $1.39 \pm 0.36$ & $1.2 \pm 0.28$ & $1.39 \pm 0.36$ & $1.2 \pm 0.28$ & $1.74 \pm 0.39$ & $1.52 \pm 0.36$ \\
\hline Total cholesterol (mmol/L) & $4.87 \pm 0.91$ & $4.99 \pm 0.92$ & $4.87 \pm 0.91$ & $4.99 \pm 0.92$ & $4.72 \pm 0.88$ & $5.01 \pm 0.93$ \\
\hline Serum uric acid $(\mu \mathrm{mol} / \mathrm{L})$ & $385.07 \pm 76.69$ & $416.59 \pm 80.88$ & $385.07 \pm 76.69$ & $416.59 \pm 80.88$ & $272.03 \pm 53.88$ & $303.07 \pm 65.69$ \\
\hline Creatinine $(\mu \mathrm{mol} / \mathrm{L})$ & $86.19 \pm 12.76$ & $85.37 \pm 13.55$ & $86.19 \pm 12.76$ & $85.37 \pm 13.55$ & $63.17 \pm 9.99$ & $63.58 \pm 10.74 *$ \\
\hline Blood urea nitrogen $(\mathrm{mmol} / \mathrm{L})$ & $5.22 \pm 1.3$ & $5.27 \pm 1.29$ & $5.22 \pm 1.3$ & $5.27 \pm 1.29$ & $4.65 \pm 1.2$ & $4.98 \pm 1.29$ \\
\hline Alanine aminotransferase (IU/L) & $30.06 \pm 19.74$ & $40.31 \pm 25.48$ & $30.06 \pm 19.74$ & $40.31 \pm 25.48$ & $18.11 \pm 11.8$ & $24.42 \pm 16.52$ \\
\hline Aspartate aminotransferase (IU/L) & $154.91 \pm 18.41$ & $155.98 \pm 19.67$ & $154.91 \pm 18.41$ & $155.98 \pm 19.67$ & $132.3 \pm 15.83$ & $133.87 \pm 15.34$ \\
\hline$\gamma$-Glutamyltransferase (IU/L) & $37.09 \pm 51.54$ & $55.73 \pm 63.99$ & $37.09 \pm 51.54$ & $55.73 \pm 63.99$ & $17.07 \pm 19.47$ & $25.98 \pm 28.57$ \\
\hline Smoking $(\%)$ & 31.30 & 37.90 & 52.70 & 56.40 & 1.60 & $1.80 *$ \\
\hline Alcohol use (\%) & 45.90 & 53.90 & 72.80 & 76.80 & 8.70 & $9.50 *$ \\
\hline Hypertension (\%) & 5.40 & 14.90 & 6.90 & 15.60 & 3.20 & 13.60 \\
\hline Diabetes mellitus (\%) & 2.40 & 5.10 & 3.30 & 5.60 & 1.10 & 4.20 \\
\hline
\end{tabular}

$* p>0.05$ 
Table 3 Comparison of clinical and demographic characteristics between $H$. pylori-positive and $H$. pylori-negative subjects

\begin{tabular}{|c|c|c|c|c|c|c|}
\hline & \multicolumn{2}{|l|}{ Total $n=76,915$} & \multicolumn{2}{|l|}{ Male $n=46,003$} & \multicolumn{2}{|l|}{ Female $n=30,912$} \\
\hline & $\begin{array}{l}\text { H. pylori-negative } \\
n=46,189\end{array}$ & $\begin{array}{l}\text { H. pylori-positive } \\
n=30,726\end{array}$ & $\begin{array}{l}\text { H. pylori-negative } \\
n=27,444\end{array}$ & $\begin{array}{l}\text { H. pylori-positive } \\
n=18,559\end{array}$ & $\begin{array}{l}\text { H. pylori-negative } \\
n=18,745\end{array}$ & $\begin{array}{l}\text { H. pylori-positive } \\
n=12,167\end{array}$ \\
\hline Age (years) & $43.8 \pm 12.67$ & $45.21 \pm 12.22$ & $44.69 \pm 12.64$ & $45.92 \pm 12.3$ & $42.49 \pm 12.59$ & $44.13 \pm 12.01$ \\
\hline Height (cm) & $163.54 \pm 7.88$ & $163.5 \pm 7.82 *$ & $167.9 \pm 6.06$ & $167.73 \pm 5.98$ & $157.16 \pm 5.55$ & $157.03 \pm 5.52$ \\
\hline $\mathrm{BMI}\left(\mathrm{kg} / \mathrm{m}^{2}\right)$ & $23.63 \pm 3.28$ & $23.83 \pm 3.31$ & $24.74 \pm 3.05$ & $24.9 \pm 3.08$ & $22.01 \pm 2.92$ & $22.19 \pm 2.95$ \\
\hline $\mathrm{WC}(\mathrm{cm})$ & $80.49 \pm 10.02$ & $81.16 \pm 10.03$ & $85.16 \pm 8.3$ & $85.7 \pm 8.32$ & $73.65 \pm 8.24$ & $74.23 \pm 8.29$ \\
\hline Hip circumference (cm) & $93.62 \pm 6.02$ & $93.84 \pm 6.05$ & $95.47 \pm 5.7$ & $95.59 \pm 5.75$ & $90.92 \pm 5.43$ & $91.18 \pm 5.51$ \\
\hline SBP (mmHg) & $113.48 \pm 15.61$ & $114 \pm 15.77$ & $116.53 \pm 15.01$ & $117.12 \pm 15.12$ & $109.03 \pm 15.41$ & $109.23 \pm 15.55^{*}$ \\
\hline DBP (mmHg) & $74.15 \pm 9.98$ & $74.55 \pm 10.13$ & $76.51 \pm 9.98$ & $76.98 \pm 10.04$ & $70.7 \pm 8.93$ & $70.83 \pm 9.09 *$ \\
\hline Pulse pressure (mmHg) & $39.33 \pm 10.94$ & $39.45 \pm 10.95 *$ & $40.02 \pm 10.95$ & $40.14 \pm 10.9^{*}$ & $38.32 \pm 10.85$ & $38.41 \pm 10.93 *$ \\
\hline FBG $(\mathrm{mmol} / \mathrm{L})$ & $5.31 \pm 1.14$ & $5.35 \pm 1.22$ & $5.44 \pm 1.3$ & $5.48 \pm 1.41$ & $5.14 \pm 0.8$ & $5.16 \pm 0.83$ \\
\hline TG (mmol/L) & $1.62 \pm 1.34$ & $1.65 \pm 1.38$ & $1.91 \pm 1.53$ & $1.93 \pm 1.55^{*}$ & $1.2 \pm 0.83$ & $1.22 \pm 0.92$ \\
\hline LDL-cholesterol (mmol/L) & $2.82 \pm 0.78$ & $2.86 \pm 0.78$ & $2.92 \pm 0.78$ & $2.95 \pm 0.78$ & $2.67 \pm 0.76$ & $2.74 \pm 0.77$ \\
\hline HDL cholesterol (mmol/L) & $1.49 \pm 0.4$ & $1.47 \pm 0.41$ & $1.35 \pm 0.35$ & $1.33 \pm 0.35$ & $1.7 \pm 0.39$ & $1.69 \pm 0.39^{*}$ \\
\hline Total cholesterol (mmol/L) & $4.82 \pm 0.91$ & $4.86 \pm 0.91$ & $4.89 \pm 0.91$ & $4.91 \pm 0.92$ & $4.72 \pm 0.9$ & $4.79 \pm 0.9$ \\
\hline Serum uric acid $(\mu \mathrm{mol} / \mathrm{L})$ & $347.35 \pm 91.18$ & $349.39 \pm 91.38$ & $394.43 \pm 78.55$ & $395.06 \pm 79.41$ & $278.42 \pm 58.77$ & $279.72 \pm 58.58^{*}$ \\
\hline Creatinine $(\mu \mathrm{mol} / \mathrm{L})$ & $76.48 \pm 16.13$ & $76.96 \pm 16.32$ & $85.62 \pm 12.68$ & $85.93 \pm 13.02$ & $63.09 \pm 10.21$ & $63.27 \pm 10.18^{*}$ \\
\hline Blood urea nitrogen $(\mathrm{mmol} / \mathrm{L})$ & $5.02 \pm 1.3$ & $5.04 \pm 1.3^{*}$ & $5.24 \pm 1.29$ & $5.24 \pm 1.3^{*}$ & $4.71 \pm 1.24$ & $4.73 \pm 1.23 *$ \\
\hline ALT (IU/L) & $27.73 \pm 20.75$ & $27.91 \pm 20.36^{*}$ & $33.65 \pm 22.89$ & $33.31 \pm 22.24 *$ & $19.05 \pm 12.91$ & $19.67 \pm 13.4$ \\
\hline AST (IU/L) & $146.57 \pm 20.43$ & $146.32 \pm 20.83^{*}$ & $155.68 \pm 18.47$ & $155.25 \pm 18.83$ & $133.22 \pm 15.14$ & $132.69 \pm 15.72$ \\
\hline$\gamma$-GGT (IU/L) & $33.11 \pm 45.17$ & $33.6 \pm 47.53^{*}$ & $42.82 \pm 52.69$ & $43 \pm 56.46^{*}$ & $18.9 \pm 24.94$ & $19.28 \pm 22.41 *$ \\
\hline Central obesity (\%) & 26.40 & 28.90 & 59.70 & 40.30 & 21.90 & 24.80 \\
\hline Smoking (\%) & 31.30 & 33.20 & 51.80 & 53.90 & 1.30 & 1.70 \\
\hline Alcohol use (\%) & 4.20 & 48.20 & 73.90 & $74 *$ & 8.10 & 8.90 \\
\hline Hypertension (\%) & 7.50 & 8.10 & 8.80 & 9.70 & 5.50 & $5.80^{*}$ \\
\hline Diabetes mellitus (\%) & 2.70 & 3.20 & 3.60 & 4 & 1.35 & 1.80 \\
\hline
\end{tabular}

${ }^{*} p>0.05$

$42.05 \%$ in quintile 2,3 , and 4 , respectively ( $p$ for trend $<0.001)$. These results in Table 4 showed that individuals with a higher WC had an increased $H$. pylori infection risk.

As shown in Table 5, after adjusting for age, gender, HC, SBP, DBP, FBG, TG, LDL-C, HDL-C, TC, UA, Scr, smoking, alcohol use, hypertension, and diabetes mellitus, central obesity was still found to be positively correlated with $H$. pylori infection $(\mathrm{OR}=1.052,95 \% \mathrm{CI}, 1.009-1.096, p=0.02)$.
As shown in Tables 6 and 7, after adjusting for age, gender, HC, SBP, DBP, FBG, TG, LDL-C, HDL-C, TC, UA, Scr, smoking, alcohol use, hypertension, and diabetes mellitus, age, central obesity, SBP, hypertension, diabetes mellitus, alcohol use were associated with $H$. pylori infection in female, while age, central obesity, SBP, DBP,TG, HDL cholesterol, and smoking were associated with $H$. pylori infection in male.

Central obesity was still found to be positively correlated with H. pylori infection in male (OR $=1.049,95 \%$ CI, $1.004-$
Table 4 Association of WC with prevalence rate of Helicobacter pylori infection

\begin{tabular}{lllllll}
\hline WC & Total & $\begin{array}{l}\text { H.pylori } \\
\text { infection }\end{array}$ & $\begin{array}{l}\text { H.pylori } \\
\text { prevalence rate (\%) }\end{array}$ & $\begin{array}{l}\text { H.pylori prevalence } \\
\text { ratio }\end{array}$ & $\chi^{2}$ & $p$ value \\
\hline Quintile 1 & 20,044 & 7569 & 37.76 & 1.00 & 79.04 & 0.000 \\
Quintile 2 & 19,710 & 7782 & 39.48 & 1.05 & & \\
Quintile 3 & 19,576 & 7981 & 40.77 & 1.08 & & \\
Quintile 4 & 17,585 & 7394 & 42.05 & 1.11 & \\
\hline
\end{tabular}


Table 5 Analysis of the relationship between $H$. pylori infection and central obesity: univariate and multivariate analysis

\begin{tabular}{|c|c|c|c|c|c|c|}
\hline & \multicolumn{3}{|c|}{ Unadjusted } & \multicolumn{3}{|c|}{ Adjusted } \\
\hline & $p$ value & OR & $95 \% \mathrm{CI}$ & $p$ value & OR & $95 \% \mathrm{CI}$ \\
\hline Age & 0.00 & 1.009 & $1.008-1.010$ & 0.00 & 1.010 & $1.009-1.011$ \\
\hline Gender & 0.01 & 0.960 & $0.932-0.989$ & 0.00 & 1.126 & $1.066-1.19$ \\
\hline Central obesity & 0.00 & 1.138 & $1.102-1.175$ & 0.02 & 1.052 & $1.009-1.096$ \\
\hline Systolic blood pressure & 0.00 & 1.002 & $1.001-1.003$ & 0.00 & 0.996 & $0.995-0.997$ \\
\hline Diastolic blood pressure & 0.00 & 1.004 & $1.002-1.005$ & 0.00 & 1.005 & $1.003-1.007$ \\
\hline Triglycerides & 0.01 & 1.015 & $1.004-1.026$ & 0.02 & 0.971 & $0.948-0.995$ \\
\hline HDL cholesterol & 0.00 & 0.888 & $0.857-0.921$ & 0.00 & 0.844 & $0.784-0.907$ \\
\hline Smoking & 0.00 & 1.091 & $1.058-1.125$ & 0.00 & 1.083 & $1.043-1.125$ \\
\hline Alcohol use & 0.01 & 1.041 & $1.012-1.072$ & 0.02 & 1.048 & $1.008-1.09$ \\
\hline Hypertension & 0.00 & 1.096 & $1.039-1.156$ & 0.07 & 0.946 & $0.892-1.004$ \\
\hline Diabetes mellitus & 0.00 & 1.069 & $1.026-1.113$ & 0.02 & 1.050 & $1.007-1.095$ \\
\hline
\end{tabular}

$O R$ odds ratio, $C I$ confidence interval
$1.096, p=0.032)$ and female $(\mathrm{OR}=1.075,95 \% \mathrm{CI}, 1.011-$ $1.143, p=0.02)$, respectively.

\section{Discussion}

Our study found that the prevalence of $H$. pylori infection was significantly higher in subjects with central obesity $(42.20 \%)$ compared with that in normal-WC subjects $(39.10 \%)$. Similarly, a small-sample study of 103 obese subjects by Arslan et al [19] reported that the prevalence of $H$. pylori infection was greater in obese subjects (57.2\%) versus the control group (27\%). An association between central obesity and $H$. pylori infection was observed in our study, which was also consistent with previous findings. A study involving 2913 Danish adults [20] found that the seroprevalence of H. pylori infection was increased in persons with high BMI. Another study from Iceland [21] also revealed that $H$. pylori infection was associated with overweight. Moreover, several studies [3, 8-10, 22] based on Chinese populations have also drawn a similar conclusion that obesity is linked to $H$. pylori infection. A study conducted in Central China indicated that

Table 6 Multivariate analysis of the relationship between H. pylori infection and central obesity in female

\begin{tabular}{llll}
\hline & $p$ & OR & $95 \%$ CI \\
\hline Age (years) & 0.000 & 1.013 & $1.011-1.016$ \\
Central obesity & 0.020 & 1.075 & $1.011-1.143$ \\
SBP (mmHg) & 0.000 & 0.994 & $0.992-0.996$ \\
Hypertension & 0.031 & 0.885 & $0.792-0.989$ \\
Diabetes mellitus & 0.015 & 1.288 & $1.051-1.578$ \\
Alcohol use & 0.024 & 1.102 & $1.013-1.199$ \\
\hline
\end{tabular}

the prevalence of $H$. pylori infection increased with BMI. Yang et al [10] showed that $H$. pylori infection confirmed by gastric biopsy pathology was associated with obesity assessed by BMI in an elderly Chinese population. Guo et al. [9] reported that waist circumference played an important role in H. pylori infection which was detected by serous $H$. pylori IgG antibodies; and the vacuolating cytotoxin gene A (VacA) was the most common strain in Northern China. However, some studies found that H. pylori infection had no association with BMI. A case-control study [23] in patients with morbid obesity from Taiwan revealed an opposite connection between morbid obesity and $H$. pylori seropositivity. A study [24] including 370 severely obese patients showed that the BMI of $H$. pylori-positive subjects did not differ from that of $H$. pylori-negative subjects. Ioannou et al. [25] noted that the status of $H$. pylori/CagA antibody was not related with obesity. A study from Greece [26] revealed that the risk of H. pylori infection was not increased among obese young individuals. Another study [27] consisting of 801 healthy university students showed that there was no significant difference in the BMI between subjects with and without $H$. pylori

Table 7 Multivariate analysis of the relationship between $H$. pylori infection and central obesity in male

\begin{tabular}{llll}
\hline & $p$ & OR & $95 \% \mathrm{CI}$ \\
\hline Age (years) & 0.000 & 1.009 & $1.007-1.011$ \\
Central obesity & 0.032 & 1.049 & $1.004-1.096$ \\
SBP (mmHg) & 0.024 & 0.998 & $0.996-1.000$ \\
DBP (mmHg) & 0.000 & 1.006 & $1.003-1.008$ \\
TG (mmol/L) & 0.005 & 0.961 & $0.934-0.988$ \\
HDL cholesterol (mmol/L) & 0.000 & 0.762 & $0.695-0.835$ \\
Smoking & 0.000 & 1.082 & $1.041-1.124$ \\
\hline
\end{tabular}


infection based on the presence or absence of specific $\operatorname{IgG}$ H. pylori antibodies in urine. But our study has shown that the BMI and $\mathrm{WC}$ in H. pylori-positive subjects were higher than in H. pylori-negative subjects $(p<0.05)$ and central obesity is associated with $H$. pylori infection after adjusting for multiple confounding factors such as age, gender, and lifestyle characteristics. On the one hand, obesity is known to increase the risk of infections [28]. Immune cell dysfunction due to obesity may result in weakened host defense [29]. Many studies in animal models and human subjects have shown that obesity can change both innate and adaptive immunity. Excess adiposity is associated with impaired immune response. Central obesity may be a predisposing factor to H. pylori infection. On the other hand, as virus can lead to infectobesity, H. pylori may be a contributing factor to obesity. The virus induces obesity probably by means of interacting with the hypothalamic-pituitary-adrenal axis [30], while the possible mechanisms of $H$. pylori-induced obesity include interruption of leptin secretion, glucose uptake, and inflammation [31], H. pylori infection can induce chronic low-grade inflammation, and then prolonged, low-level immune stimulation induces hypertrophy of adipose tissue and increases shunting of energy to host defense mechanisms [32]. Our study found that the FPG, TG, LDL-C, and TC levels were higher in H. pylori-positive subjects than those in H. pylorinegative subjects. Thus, we speculate that $H$. pylori infection contributes to obesity by disrupting glycometabolism and lipid metabolism.

These conflicting findings may be explained by differences in the diagnostic methods of $H$. pylori infection, sample size, target populations, ethnic groups, geographic regions, and H. pylori strains. Most of these published studies diagnosed H. pylori infection by serology which has a low diagnostic sensitivity (85\%) and specificity (79\%), only useful to rule out H. pylori infection. The urine H. pylori antibody test [33] only detects exposure status rather than active infection, with a sensitivity of $86 \%$ and a specificity of $91 \%$. In our study, H. pylori infection was defined by ${ }^{14} \mathrm{C}-\mathrm{UBT}$, the most reliable noninvasive method that detects active $H$. pylori infection with a sensitivity of $96 \%$ and a specificity of $93 \%$.

In terms of sample size and target population, most prior studies only enrolled hundreds of or no more than ten thousand participants, but in our study, a large population of 76,915 individuals (46,003 men and 30,912 women) with a medium (interquartile ranges) age of 44.0 (35.0-51.0) years were recruited. We focused on the adult population in West China, while some other studies [34] targeted at children and adolescents. It has been shown that race, geographic region and $H$. pylori strain may be important factors affecting the association between obesity and $H$. pylori infection. A US study [25] found that the prevalence of $H$. pylori infection and CagA strains differed among non-Hispanic blacks, whites, and Mexican-Americans.
One of the strengths of our study is that a large population consisting of 76,915 subjects from West China was included in this cross-sectional retrospective survey. All of these participants underwent ${ }^{14} \mathrm{C}$-UBT and we controlled for multiple covariates to explore the association between obesity and $H$. pylori infection. Nevertheless, there are also several limitations of our study. Firstly, as this is a cross-sectional retrospective study, the casual relationship between $H$. pylori infection and central obesity cannot be assessed. Secondly, due to the very small but calculable dose of radiation, ${ }^{14} \mathrm{C}$-UBT is not appropriate for use in children [35]. Consequently, data on children is not available in the present study, and our findings could not be generalized to the entire population. Thirdly, the H. pylori strain was not determined in this study, and thus the association between different $H$. pylori strains and obesity cannot be explored.

\section{Conclusion}

Our study based on a large Chinese population in West China reveals that central obesity is associated with $\mathrm{H}$. pylori infection after adjusting for multiple confounding factors such as age, gender, and lifestyle characteristics.

Acknowledgments Qinqin Wu thanks the support from the staff members in Health Management Center, West China Hospital, Sichuan University for their generous time and support.

Contributorship statement Qinqin Wu designed the study, carried out the study, analyzed the results, and contributed to the discussion of results and drafting of the manuscript. Ken Qin analyzed and interpreted the data in the revised version. Youjuan Wang contributed to designing the study and discussion of results, and the final manuscript. All authors have read and approved the final manuscript.

Funding This work was supported by a grant from the Sichuan province health department (Grant No. Chuanganyan2012-111), the Youth Teacher Research Startup Fund of Sichuan University (2016SCU11016), and the Department of Science and Technology of Sichuan Province (Grant No. 2017RZ0046).

Data availability The data used in this study were collected from the Health Management Center of West China Hospital. These data are not publicly obtainable.

\section{Compliance with ethical standards}

Competing interests The authors declare that they have no competing interests.

Ethics approval and consent to participate This study was approved by the Ethics Committee of West China Hospital of Sichuan University. As this is a retrospective study, informed consent was not essential in line with the Ethical Guidelines for Epidemiological Research.

The study was permitted by the Ethics Committee of West China, Sichuan University.

Consent for publication Not applicable. 
Open Access This article is distributed under the terms of the Creative Commons Attribution 4.0 International License (http:// creativecommons.org/licenses/by/4.0/), which permits unrestricted use, distribution, and reproduction in any medium, provided you give appropriate credit to the original author(s) and the source, provide a link to the Creative Commons license, and indicate if changes were made.

\section{References}

1. Upala S, Sanguankeo A, Saleem SA, Jaruvongvanich V. Effects of Helicobacter pylori eradication on insulin resistance and metabolic parameters: a systematic review and meta-analysis. Eur J Gastroenterol Hepatol. 2017;29(2):153-9. https://doi.org/10.1097/ meg.0000000000000774.

2. Xu M-Y, Liu L, Yuan B-S, Yin J, Lu Q-B. Association of obesity with Helicobacter pylori infection: a retrospective study. World $\mathrm{J}$ Gastroenterol. 2017;23(15):2750-6. https://doi.org/10.3748/wjg. v23.i15.2750.

3. Zhang Y, Du T, Chen X, Yu X, Tu L, Zhang C. Association between Helicobacter pylori infection and overweight or obesity in a Chinese population. J Infect Dev Ctries. 2015;9(9):945-53. https://doi.org/10.3855/jidc.6035.

4. Vo HD, Goli S, Gill R, Anderson V, Stefanov DG, Xu J, et al. Inverse correlation between helicobacter pylori colonization and obesity in a cohort of Inner City children. Helicobacter. 2015;20(1):64-8. https://doi.org/10.1111/hel.12154.

5. Rocco A, Sgamato C, Guarracino M, Martino A, Costagliola N, Occhipinti V, et al. Helicobacter pylori infection and obesity: is there a link? Dig Liver Dis. 2014;46:S15-S6.

6. Abadi ATB, Lee YY. Letter: inverse correlation between Helicobacter pylori and obesity - a conclusion too early? Aliment Pharmacol Ther. 2014;40(9):1119. https://doi.org/10.1111/apt.12951.

7. Lender N, Talley NJ, Enck P, Haag S, Zipfel S, Morrison M, et al. Review article: associations between Helicobacter pylori and obesity - an ecological study. Aliment Pharmacol Ther. 2014;40(1):2431. https://doi.org/10.1111/apt.12790.

8. Xu CF, Yan M, Sun Y, Joo J, Wan XY, Yu CH, et al. Prevalence of helicobacter pylori infection and its relation with body mass index in a Chinese population. Helicobacter. 2014;19(6):437-42. https:// doi.org/10.1111/hel.12153.

9. Guo X, Zhao BH, Zhang MX. Risk factors of Helicobacter pylori infection among adults in northern China. Hepatogastroenterology. 2011;58(106):306-10.

10. Yang GH, Wu JS, Yang YC, Huang YH, Lu FH, Chang CJ. Obesity associated with increased risk of gastric Helicobacter pylori infection in an elderly Chinese population. J Am Geriatr Soc. 2014;62(1):190-2.

11. Kopacova M, Koupil I, Seifert B, Fendrichova MS, Spirkova J, Vorisek V, et al. Blood pressure and stature in Helicobacter pylori positive and negative persons. World J Gastroenterol. 2014;20(19): 5625-31. https://doi.org/10.3748/wjg.v20.i19.5625.

12. Danesh J, Peto R. Risk factors for coronary heart disease and infection with Helicobacter pylori: meta-analysis of 18 studies. BMJ. 1998;316(7138):1130-2.

13. Misigoj-Durakovic M, Soric M, Durakovic Z. Anthropometry in cardio-metabolic risk assessment. Arh Hig Rada Toksikol. 2014;65(1):19-27. https://doi.org/10.2478/10004-1254-65-2014-2381.

14. Burkhauser RV, Cawley J. Adding biomeasures relating to fatness and obesity to the Panel Study of Income Dynamics. Biodemography Soc Biol. 2009;55(2):118-39. https://doi.org/10. 1080/19485560903382395.
15. Ferwana M, Abdulmajeed I, Alhajiahmed A, Madani W, Firwana B, Hasan R, et al. Accuracy of urea breath test in Helicobacter pylori infection: meta-analysis. World J Gastroenterol. 2015;21(4):1305-14. https://doi.org/10.3748/wjg.v21.i4.1305.

16. Ness-Abramof R, Apovian CM. Waist circumference measurement in clinical practice. Nutr Clin Pract. 2008;23(4):397-404. https:// doi.org/10.1177/0884533608321700.

17. Alberti KG, Zimmet P, Shaw J. Metabolic syndrome-a new worldwide definition. A consensus statement from the International Diabetes Federation. Diabet Med. 2006;23(5):469-80. https://doi. org/10.1111/j.1464-5491.2006.01858.x.

18. Friedewald WT, Levy RI, Fredrickson DS. Estimation of the concentration of low-density lipoprotein cholesterol in plasma, without use of the preparative ultracentrifuge. Clin Chem. 1972;18(6):499-502.

19. Arslan E, Atilgan H, Yavasoglu I. The prevalence of Helicobacter pylori in obese subjects. Eur J Intern Med. 2009;20(7):695-7. https://doi.org/10.1016/j.ejim.2009.07.013.

20. Rosenstock SJ, Jorgensen T, Andersen LP, Bonnevie O. Association of Helicobacter pylori infection with lifestyle, chronic disease, body-indices, and age at menarche in Danish adults. Scand J Public Health. 2000;28(1):32-40.

21. Thjodleifsson B, Olafsson I, Gislason D, Gislason T, Jogi R, Janson C. Infections and obesity: a multinational epidemiological study. Scand J Infect Dis. 2008;40(5):381-6. https://doi.org/10.1080/ 00365540701708293.

22. Chen TPHH, Chen MK, Lai HH, Hsu WF, Huang KC, Yang KC. Helicobacter pylori infection is positively associated with metabolic syndrome in Taiwanese adults: a cross-sectional study. Helicobacter. 2015;20(3):184-91.

23. Wu MS, Lee WJ, Wang HH, Huang SP, Lin JT. A case-control study of association of Helicobacter pylori infection with morbid obesity in Taiwan. Arch Intern Med. 2005;165(13): 1552-5. https://doi.org/10.1001/archinte.165.13.1552.

24. Gerig R, Ernst B, Wilms B, Thurnheer M, Schultes B. Gastric Helicobacter pylori infection is associated with adverse metabolic traits in severely obese subjects. Obesity (Silver Spring). 2013;21(3):535-7. https://doi.org/10.1002/oby.20098.

25. Ioannou GN, Weiss NS, Kearney DJ. Is Helicobacter pylori seropositivity related to body mass index in the United States? Aliment Pharmacol Ther. 2005;21(6):765-72. https://doi.org/10.1111/j. 1365-2036.2005.02369.x.

26. Kyriazanos ID, Sfiniadakis I, Gizaris V, Hountis P, Hatziveis K, Dafnopoulou A, et al. The incidence of Helicobacter pylori infection is not increased among obese young individuals in Greece. J Clin Gastroenterol. 2002;34(5):541-6.

27. Shiotani A, Miyanishi T, Uedo N, Iishi H. Helicobacter pylori infection is associated with reduced circulating ghrelin levels independent of body mass index. Helicobacter. 2005;10(5):373-8. https://doi.org/10.1111/j.1523-5378.2005.00343.x.

28. Genoni G, Prodam F, Marolda A, Giglione E, Demarchi I, Bellone $\mathrm{S}$, et al. Obesity and infection: two sides of one coin. Eur J Pediatr. 2014;173(1):25-32. https://doi.org/10.1007/s00431-013-2178-1.

29. Hegde V, Dhurandhar NV. Microbes and obesity-interrelationship between infection, adipose tissue and the immune system. Clin Microbiol Infect. 2013;19(4):314-20. https://doi.org/10.1111/ 1469-0691.12157.

30. Falagas ME, Kompoti M. Obesity and infection. Lancet Infect Dis. 2006;6(7):438-46. https://doi.org/10.1016/s1473-3099(06)70523-0.

31. Na H. Infectobesity: a new area for microbiological and virological research. J Bacteriol Virol. 2011;41(2):65-76.

32. Khosravi Y, Seow SW, Amoyo AA, Chiow KH, Tan TL, Wong WY, et al. Helicobacter pylori infection can affect energy modulating hormones and body weight in germ free mice. Sci Rep. 2015;5: 8731. https://doi.org/10.1038/srep08731. 
33. Mabe K, Kikuchi S, Okuda M, Takamasa M, Kato M, Asaka M. Diagnostic accuracy of urine Helicobacter pylori antibody test in junior and senior high school students in Japan. Helicobacter. 2017;22(1). https://doi.org/10.1111/ hel.12329.

34. Han X, Li Y, Wang J, Liu B, Hu H, Li X, et al. Helicobacter pylori infection is associated with type 2 diabetes among a middle- and old-age Chinese population. Diabetes Metab Res Rev. 2016;32(1): 95-101. https://doi.org/10.1002/dmrr.2677.
35. Geng L, Gong S. Paying attention to the diagnosis and treatment of Helicobacter pylori infection in children. Chin J Pract Pediatr. 2016;31(7):490-3.

Publisher's note Springer Nature remains neutral with regard to jurisdictional claims in published maps and institutional affiliations. 